\title{
An oscillation effect at 5 seconds' duration of stimulation
}

\author{
JOHN GAITO \\ York University, 4700 Keele Street, Downsview, Ontario M3J 1P3, Canada
}

\begin{abstract}
Previous research indicated that an oscillation effect resulted during sequential alternation of unilateral amygdaloid stimulation with consistently low latency values for one side and consistently high values for the contralateral one when $30 \mathrm{sec}$ was the duration of stimulation. In the present experiment, stimulation was for $5 \mathrm{sec}$. The oscillation tendency was the same with 5 sec duration as it had been with $30 \mathrm{sec}$.
\end{abstract}

The "kindling effect" has been investigated in a number of laboratories as a model of learning, a model of epilepsy, or as an example of behavioral change of interest in its own right (e.g., Gaito \& Gaito, 1974; Goddard, McIntyre, \& Leech, 1969). This event involves a change from normal exploration to automatic behaviors and, finally, to clonic convulsions in response to electrical stimulation of a specific brain site (e.g., amygdala).

In recent research in my laboratory, using a sequence of alternating unilateral stimulation of the amygdalae, an "oscillation effect" was observed (Gaito, 1976). This oscillation (or fluctuation) usually consisted of low values for the amygdala first stimulated (primary site) and high values for the contralateral amygdala (secondary site). The effect was most prominent in latency data (number of seconds from onset of stimulation to onset of convulsion). The oscillation tendency in latency data has been remarkably resistant to a number of experimental manipulations (Gaito, Gaito, \& Nobrega, 1977). It is assumed to be due to transfer and interference effects between the two amygdalae (Gaito, Nobrega, \& Gaito, 1978; McIntyre \& Goddard, 1973; Nobrega \& Gaito, 1978).

The previous experiments had used $30 \mathrm{sec}$ of stimulation. It is possible that this long duration of stimulation contributed to the oscillation tendency. In the present study, a shorter duration of stimulation $(5 \mathrm{sec})$ was investigated.

\section{METHOD}

Twelve male Wistar rats (approximately 165 days of age) were implanted bilaterally with bipolar electrodes in each amygdala. The brain coordinates for electrode implantation were the same as in previous experiments: $.5 \mathrm{~mm}$ posterior to bregma, $4.5 \mathrm{~mm}$ from midline, $8.5 \mathrm{~mm}$ from skull (Gaito, 1976; Gaito \& Gaito, 1974).

Stimulation trials began a week or more after electrode implantation. Each trial consisted of passing a $60-\mathrm{Hz}$ sine wave for $5 \mathrm{sec}$ into the rat using a Lafayette stimulator; there were three trials per day with approximately $1 \mathrm{~h}$ between trials. The

This research was supported by a grant from the President's NRC Fund (York University). initial intensity was set at 100 microA for all animals; the current was gradually increased in the cases of no clonic convulsion (CC) by 10 trials until the appropriate intensity was achieved for each animal. In most cases, the convulsion was achieved with 100 microA and in no case was the intensity level raised beyond 580 microA.

After six CCs were achieved at the primary site, a similar procedure was followed with the secondary site in Phase 2 until six CCs occurred. This alternation from primary to secondary site continued for 10 phases.

On the first trial of Phases 3 and 4, the intensity was slowly increased from subthreshold values until a definite response occurred. Then the current was increased approximately 15 microA beyond this point to handle day by day fluctuations. The first CC trial in each of these phases was not used in the data analyses, because varying amounts of time were required in finding intensities that were just above threshold for each side.

For each rat the behavioral designation for the latency and criterion measures was that of primary oscillator, secondary oscillator, or nonoscillator. For exämple, to be classified as an oscillator, the rat had to show a consistent pattern of "low-high" (primary oscillation) or "high-low" (secondary oscillation) in latency and/or criterion data over 8 or 10 of the 10 alternation phases. As in the previous research, duration values were not analyzed.

\section{RESULTS AND DISCUSSION}

Three of the 12 rats lost electrodes after five or six phases. In latency data, six of the rats that completed 10 phases showed an oscillation pattern; three did not. Primary oscillation (low values for primary site) was present in four of the six rats. Two rats had the opposite pattern (secondary oscillation, low values for secondary site). The finding of six oscillators out of nine rats was significantly different from the number expected by chance, using a $\mathrm{p}$ of oscillation of $.17 .^{1}$ Thus, the latency results were consistent with those of previous research.

In criterion data only three of the nine rats had an oscillation pattern (two with primary oscillation, one with secondary oscillation). These results were not significantly different from those expected by chance. Seldom does significance occur with the criterion measure. 
These results were consistent in both latency and criterion measures with those of previous research using $30 \mathrm{sec}$ as the duration of stimulation. Thus, a 30-sec duration of stimulation is not required for oscillation to occur.

These results are also consistent with those of McIntyre and Goddard (1973). These individuals used sequential alternation of unilateral stimulation over three phases (primary, secondary, primary). Over these three phases, a primary oscillation pattern in the latency data was observed (i.e., low, high, low). The mean values for the nine rats in the present experiment were 7.3, 10.6, and 6.7, thus being consistent with the McIntyre and Goddard results. If the values of the three rats that lost electrodes were included, the results would be similar.

In the criterion measure, the present results are similar to those of McIntyre and Goddard (1973) in that fewer trials were required to reach the criterion of six convulsions with the first stimulation of the secondary site. However, each of the nine animals in the present study convulsed on every stimulation trial with the primary site after stimulation of the secondary site (Phase 3), whereas McIntyre and Goddard found that some rats had one or more trials of nonconvulsion before the convulsions occurred. But, if the three rats that lost electrodes were included in the data, the present results would be more consistent with those of McIntyre and Goddard. These three rats required 8, 8, and 10 trials to effect six clonic convulsions.

To account for the oscillation effect in this experiment and in previous research, it is assumed that there are some inhibitory and/or facilitatory effects from the primary site to the secondary site, and vice versa (Gaito et al., 1978; Nobrega \& Gaito, 1978). However, it is not clear what the exact basis for the effect is. A pattern appears to be set up, either primary oscillation or secondary oscillation, during Phases 1 and 2, or by Phases 3 and 4, and most rats continue with this pattern for the remainder of the 10 phases. In one experiment in which rats were stimulated through 50 phases, some rats showed a consistent pattern of oscillation for the 50 phases (Gaito, 1978).

The unilateral sequential alternation procedure and the resulting oscillation effect appear to be useful for obtaining information on some aspects of the events underlying the kindling effect, for example, possible differential electrical synaptic patterns related to the difference in latency values for the primary and secondary sites. Because kindling shows many parallels to learning and to epilepsy and can be considered as a model of consistent behavioral changes in response to an invariant stimulus, experimentation with the oscillation effect may have the potential for providing information relevant to brain function in general.

\section{REFERENCES}

Garro, J. An oscillation effect during sequential alternations of unilateral amygdaloid stimulations within the kindling paradigm. Physiological Psychology, 1976, 4, 303-306.

Garto, J. The oscillation effect over long-term periods. Bulletin of the Psychonomic Society, 1978, 11, 9-12.

Garto, J., \& Garto, S. T. Interanimal negative transfer of the kindling effect. Physiological Psychology, 1974, 2, 379-382.

Gaito, J., Gatto, S. T., \& Nobrega, J. N. A factor analysis of data from 10 phases of sequential alternations of amygdaloid stimulation within the kindling paradigm. Physiological Psychology, 1977, 5, 300-310.

Garto, J., NobregA, J. N., \& Garto, S. T. Statistical evaluation of several aspects concerning the oscillation effect. Physiological Psychology, 1978, 6, 209-214.

Goddard, G. V., McINTYRe, D. C., \& LeEch, C. K. A permanent change in brain function resulting from daily electrical stimulation. Experimental Neurology, 1969, 25, 295-330.

MCINTYRE, D. C., \& GoDdARD, G. V. Transfer, interference and spontaneous recovery of convulsions kindled from the rat amygdala. Electroencephalography and Clinical Neurophysiology, 1973, 35, 533-543.

Nobrega, J. N., \& Garto, J. Long term induction of kindled seizures in rats: Interhemispheric factors. Canadian Journal of Neurological Sciences, 1978, 5, 223-230.

\section{NOTE}

1. The hypothesis that the oscillation patterns are random ones can be assessed by the one-sample runs test. Using the requirement of 8 or 10 phases of oscillation out of 10 phases would provide 8,9 , or 10 runs. The $p$ of 8,9 , and 10 runs is $.1270, .0317$, and .0079 , respectively; the $p$ of 8,9 , or 10 runs is the sum of these ps, .1666 or .17. Thus the $p$ that these apparently systematic patterns are random ones would be .17 (Gaito et al., 1978).

(Received for publication July 11, 1978.) 